【电子与信息科学／Electronics and Information Science】

\title{
面向汽车生态圈的区块链应用模型
}

\author{
陈 洁 $^{1}$, 张胜利 ${ }^{2}$, 王 晖 $^{2}$
}

1) 北京大学教育学院, 北京 100871 ;

2) 深圳大学电子与信息工程学院，人工智能与数字经济广东省实验室，广东深圳 518060

摘 要：针对汽车行业服务生态中的交易数据不透明、交易参与方缺乏互信的问题，以某汽车厂商售 后预付费产品为研究对象，应用区块链智能合约技术，构建面向汽车生态圈的去信任系统模型. 该模型首 次系统论述了在汽车行业预付费产品场景下区块链上各参与方的角色、业务流程及智能合约的实现方案, 丰富了联盟链的应用场景，可推广应用于跨行业生态服务系统.

关键词: 计算机网络; 区块链; 汽车服务生态圈; 联盟链; 智能合约; 预付费产品; 去信任系统

中图分类号：TN919.2；TP393.4 文献标志码：A doi：10.3724/SP. J.1249.2021.06643

\section{Application of blockchain technology in automobile ecosystem}

\section{CHEN Jie ${ }^{1}$, ZHANG Shengli ${ }^{2}$, and WANG Hui ${ }^{2}$}

1) Graduate School of Education, Peking University, Beijing 100871, P. R. China

2) College of Electronic and Information Engineering, Guangdong Province Lab of Artificial Intelligence and

Digital Economy, Shenzhen University, Shenzhen 518060, Guangdong Province, P. R. China

\begin{abstract}
Aiming at the challenges of opaque transaction data and lack of mutual trust among participants in the service ecosystem of the automobile industry, a de-trust system model based on blockchain smart contract technology is constructed, which takes the after-sales prepaid products from an automobile manufacturer as the research object. Under the scenario of prepaid products in automotive industry, this model systematically discusses the roles of participants, business processes and smart contract solution in the blockchain for the first time. The proposed model can not only enrich the application scenarios of consortium blockchain but also be extended to more cross industry ecosystems.
\end{abstract}

Key words : computer networks; blockchain; automobile service ecosystem; consortium blockchain; smart contract; prepaid products; de-trust system

美国汽车协会 (American Automobile Association, $\mathrm{AAA})$ 将汽车售后服务定义为汽车在售出之后 维修和保养所使用的零配件和服务 ${ }^{[1]}$, 是汽车行业 的主要获利方式 ${ }^{[2]}$. 中国目前的汽车售后市场服务 信息透明度和服务质量仍有待提升. 据报道, 在 2019 年全国消协组织受理的投诉中, 针对售后服务
的投诉达到 $29.09 \%$, 在所有投诉类型中占比最 高 $^{[3]}$, 而在具体商品投诉中, 对汽车及零部件投诉 高居投诉首位. 造成这一状况的原因主要有两方 面：(1) 车辆的保养、维修和二手车交易缺乏客户 可信赖、可查询的信息记录和记录方式. (2) 汽车 后市场服务的复杂性和高度专业性使车主无法对售

Received: 2020-12-31 ; Revised: 2021-06-02 ; Accepted : 2021-08-31 ; Online (CNKI) : 2021-10-26

Foundation: National Natural Science Foundation of China (61771315)

Corresponding author: Professor ZHANG Shengli. E-mail: zsl@ szu.edu.cn

Citation: CHEN Jie, ZHANG Shengli, WANG Hui. Application of blockchain technology in automobile ecosystem [ J]. Journal of Shenzhen University Science and Engineering, 2021, 38(6): 643-648. (in Chinese) 
后服务的质量做量化评估，导致售后服务鱼龙混 杂, 不仅降低了客户的满意度, 也令行业参与方的 互利互信面临巨大挑战. 在汽车维修保养服务方 面, 车主 (用户) 、汽车厂商和经销商 3 方利益点存 在矛盾. 车主需要高品质且低成本的服务; 厂商追 求长远的品牌口碑, 因而重视客户满意度和客户黏 性; 经销商作为售后服务的提供方，在实践中常为 追求短期高利润而损害客户权益. 上述冲突导致经 销商、客户和厂商之间忠诚度和互信度面临挑战.

为提高客户保留度和满意度, 汽车厂商纷纷推 出如保养卡等售后服务预付费产品. 预付费产品所 有者是汽车厂商, 销售方是经销商, 用户以折扣价 格一次性向经销商支付多次保养的费用. 当用户在 经销商处进行保养服务时，厂商向经销商返还该次 服务的费用, 直至预存的额度全部使用完毕. 在此 过程中, 因参与方多元, 所以流程复杂, 常会涉及 多次结算, 且操作过程全由中心节点推动, 导致车 主、汽车厂商和经销商之间存在信息不透明、追责 困难和互信度低等问题. 为此, 本研究采用区块链 技术实现汽车服务生态链信息数据共享和数据安 全, 建立新型的应用模式, 从而提升汽车的售后服 务水平.

\section{1 区块链的构建}

\section{1 区块链技术起源}

区块链是比特币的底层技术, 本质上是一个去 中心化的数据库. 自区块链技术被提出以来, 越来 越多的区块链系统以及基于区块链技术的应用系统 相继问世，并被广泛应用于商业金融、医疗卫生和 物流服务等领域.

根据部署情况和应用场景, 目前区块链主要有 私有链、公有链和联盟链 3 种.

私有链是写人权限由某个组织或机构控制的区 块链，参与区块链的节点的资格会被严格限制. 由 于参与的节点是有限和可控的, 私有链具有交易速 度快、数据安全性强、交易成本低, 以及可身份认 证等特点.

公有链向所有人开放, 每个人都能成为系统中 的一个节点, 参与区块链记账. 通过将激励机制与 加密数字验证相结合, 可实现链上所有节点对交易 的共识. 由于对交易信息的流动路径并无任何要 求, 每个节点只需要尽其最大努力传播交易即 可 $^{[4]}$. 当前公有链技术 (如许可公链) 发展迅速,
区块链应用场景大大扩展 ${ }^{[5]}$. 公有链的优点包括: (1) 能够保护用户免受开发者的影响; (2) 所有交易 数据都默认公开; (3) 访问门槛低, 任何人只要有 联网设备即可访问; (4) 能够通过社区激励机制更 好地实现大规模的协作共享等.

联盟链是由若干机构共同参与记账的区块链, 联盟成员之间通过对多中心的互信来达成共识. 联 盟链只允许系统内的成员节点对数据进行读写和发 送交易，且共同记录交易数据. 联盟链更符合商业 活动中多方对等合作与合规有序发展的要求, 因此 也更适于不同组织机构之间的交易和结算，如银行 间的转账和支付. 采用联盟链可打造一个跨机构的 生态系统并大幅提高系统的管理效率.

\section{2 区块链架构}

区块链系统由应用层、合约层、激励层、共识 层、网络层和数据层构成, 基本架构如图 1 .

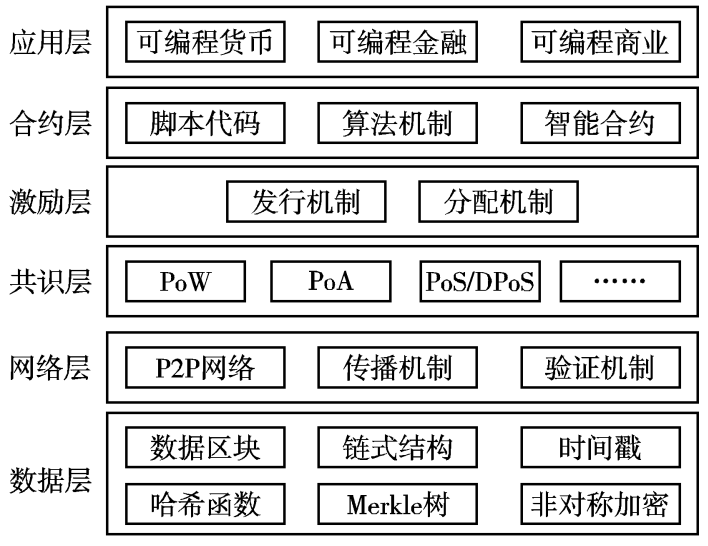

图 1 区块链基本架构 ${ }^{[6]}$

Fig. 1 Blockchain architecture ${ }^{[6]}$

1) 数据层是整个区块链系统的基础, 为区块 链系统上层功能提供支持. 区块链的每个节点存储 在本地的数据可分为交易数据、区块数据和链数据 3 个层级. 每个层级都可根据需要使用不同的加密 功能保证数据的完整性和原子性.

2）网络层主要目标是在节点间引人随机拓扑 结构，同时实现区块链更新信息的有效传播和本地 同步. 现有区块链系统一般选择去中心化的对等 ( peer-to-peer，P2P) 协议 ${ }^{[7]}$ 作为网络层, 可规避单 点故障问题.

3）共识层定义并封装了各种类型的共识协议, 确保了链上所有节点能够对区块数据合法性达成共 识. 这些共识协议包括工作量证明 ( proof of work, PoW) 共识、资产证明 (proof of authority, PoA) 共 识、权益证明/授权股份证明 ( proof of stake/delega- 
ted proof of stake, PoS/DPoS ) 共识等. 区块链中每 个节点都保存了全局唯一账本的副本, 当新区块产 生时, 各节点需及时更新本地账本. 但各节点因现 实原因并不能同时记账, 为保持账本的一致性, 需 通过竞争记账的方式选出记账节点, 由此节点广播 新的记账区块. 共识层定义的共识机制就是各节点 竞争记账权的机制.

4) 在分布式系统中, 所有节点都是自私的, 其参与区块链相关记账活动的根本目的是收人最大 化. 因此, 合理的激励兼容机制可保证分布式区块 链生态系统的安全性和有效性.

5）合约层负责封装业务逻辑代码，是区块链 系统发展的重点. 随着越来越多的脚本语言的出 现，区块链能够更好地应用于现实场景中.

6）区块链是一种底层技术，因此，应用层封 装了区块链的各种应用场景，大致可分为编程货 币，可编程金融和可编程商业 3 类. 去中心化应用 通过 Web 3.0 接口层与底层区块链系统进行交互.

以上结构中，智能合约是区块链应用系统的核 心, 借助智能合约, 可满足现实场景中各种复杂逻 辑的需求.

\section{3 智能合约}

智能合约是区块链上运行的计算机程序, 可在 满足其源代码中的写人条件时自动执行, 如图 2 . 由于合约条款不能被改变, 因此, 智能合约一旦编 写好就可被用户信赖.

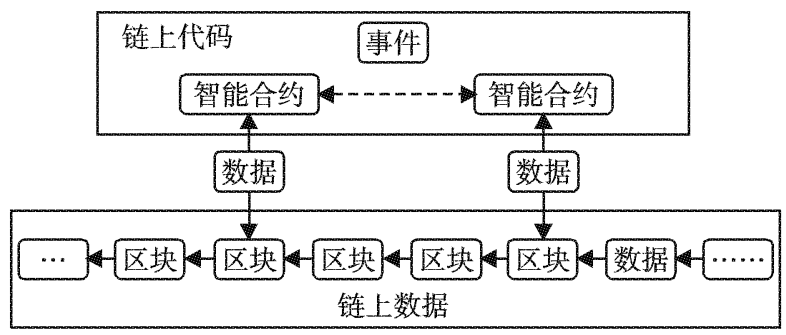

图 2 运行在区块链上的智能合约

Fig. 2 Smart contract on blockchain

资产管理智能合约是根据事先任意制订的规则 自动转移数字资产的系统, 如图 3. 合约数据通过 Merkel 前缀树 ${ }^{[8-10]}$ 组织实现各节点数据的一致性. 例如, 一个人可能有一个存储合约, 形式为 “ $A$ 每 天最多可提现 $X$ 个币, $B$ 每天最多 $Y$ 个, $A$ 和 $B$ 一起 可以随意提取, $A$ 可以停掉 $B$ 的提现权” ${ }^{[11]}$. 这种 合约的符合逻辑的扩展就是去中心化自治组织 长期包含一个组织的资产并把组织的规则进行编码 的智能合约.

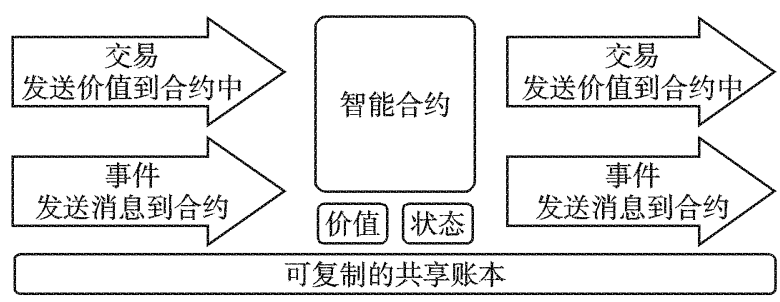

图 3 智能合约一一价值转移方式

Fig. 3 Smart contract-value transaction

2 基于区块链的汽车售后预付费产品 生态圈模型

采用区块链技术，以联盟链的形式构建起面向 汽车厂商、经销商和用户的服务生态平台. 该平台 以汽车厂商为主导方, 对汽车行业生态内的系统服 务加以整合和规范, 各服务提供商根据自身业务内 容在区块链上部署实现面向用户的服务智能合约; 用户与服务提供商签署服务产品订购相关的服务, 签约后，用户会获得服务通证（token，也称为代 币). 此 token 被视为一种服务承诺, 作为产品凭证 在整个汽车行业服务生态中进行流转, 被用来进行 支付和结算. 上述以汽车为中心、以区块链为技术 解决方案的应用模型如图 4 .

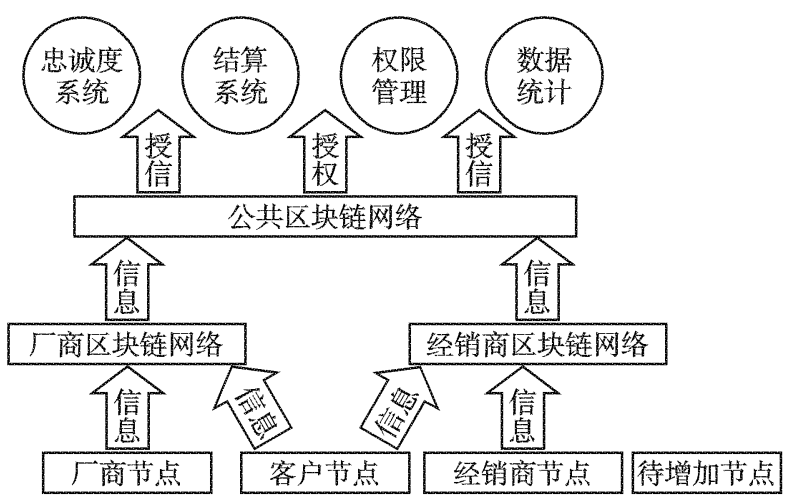

图 4 汽车联盟生态的构成

Fig. 4 Auto ecosystem structure

\section{1 系统参与方及权限}

1）超级节点即汽车厂商节点 $(M)$, 具有对全 部数据的访问权限和系统管理权限, 并保护数据安 全和系统安全.

2) 联盟节点即经销商节点 $(D)$, 具有部分数 据访问权限. 经销商的相关数据访问权限由汽车厂 商节点在注册时赋予，并提供相应的服务.

3）用户节点即应用 (application, App) 节点 $(C)$, 用于访问业务内容和发送业务信息. 用户对 
自己的全部数据有访问权.

$4)$ 待增加节点 $(E)$ 是为汽车厂商后续业务预 留的节点.

\section{2 生态圈模型业务流程}

传统汽车服务预付费产品大多是一次缴付可享 多次服务的服务储值卡，此类产品的商务和财务流 程如图 5. 该业务流程的主要弊端是资金风险. $C$ 将 未来多次服务的费用预付给 $D$, 在 $D$ 与 $M$ 结算前, 若 $D$ 发生经营风险或存在违法恶意，如破产、挪用 货款和携款潜逃等, $M$ 将无法收到货款, $C$ 未来的权 益无法得到保障，势必引发投诉. 采用区块链化的 设计改造方案, 可解决此痛点. 具体方案如下.

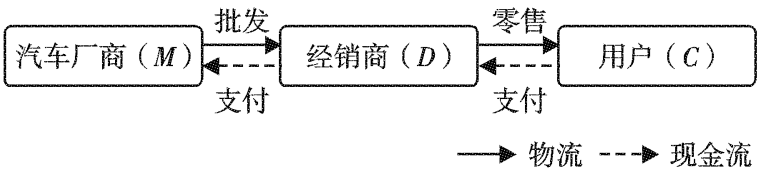

图 5 传统汽车行业物流和现金流模型

Fig. 5 Traditional goods flow and currency flow of auto industry

1）产品销售

产品销售的链上流程如图 6, 具体步骤为: (1) $C$ 用 App 发起购买 token 申请; (2) $D$ 收到申请后进 行确认; (3) $M$ 收到申请后进行确认; (4) 合约自动 发送服务产品 token 给 $C$.

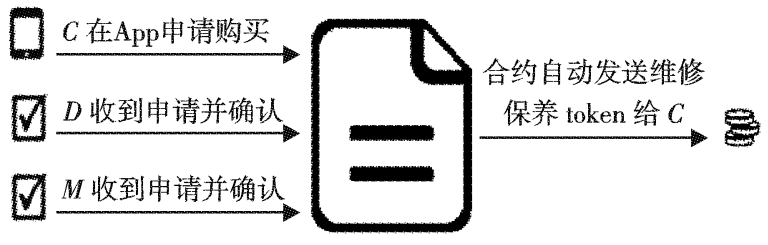

图 6 产品销售链上流程

Fig. 6 Product sales process on blockchain

产品销售的链下流程为: (1) $D$ 通过市场宣传和 促销活动等推广此项服务, 引导 $C$ 选购服务, 培养 $C$ 的购买习惯; (2) $C$ 利用 App 选购服务并付款; (3) App 利用付款凭证发起购买申请; (4) $M$ 确认此交易 生效, 自动检查并确认 $D$ 的付款记录; (5) $D$ 将收到 款项的一部分或全部转给 $M$ 作为押金.

2) 产品使用

产品使用的链上流程如图 7, 步骤为: (1) $C$ 发 送维修保养 token; (2) $D$ 确认并发送维修信息; (3) $C$ 在保养结束后, 检查车辆, 再确认并评价; (4) 将 token 保留在合约地址或 $D$ 地址.

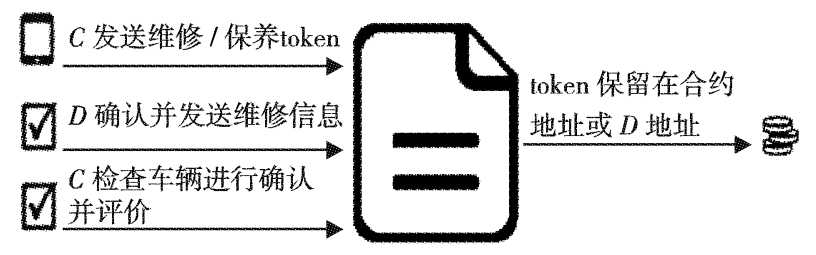

图 7 产品使用链上流程

Fig. 7 Product usage process on blockchain

产品使用的链下流程为: (1) $C$ 到 $D$ 做售后服务 (或在 App 上预约); (2) $D$ 调取车辆历史维修数据, 结合当前现状制定服务方案; (3) $D$ 根据方案进行维 修, 并将维修内容上传到区块链; (4) $C$ 在服务结束 后提交服务评价.

\section{3）服务提供商的转换}

$C$ 可在 $D_{1}$ (销售该保养卡的经销商) 和 $D_{2}$ (使用 该保养卡的经销商) 共同确认下实现 token ( $D_{1}$ 提 供) 转到 token ( $D_{2}$ 提供) 下, 则原本已购买 $D_{1}$ 服 务的 $C$ 只需在链上进行转换就可在 $D_{2}$ 使用服务.

服务提供商转换链上流程如图 8, 步骤为: (1) $C$ 使用 App 发起服务转移 token 申请; (2) $D_{1}$ 和 $D_{2}$ 确 认后允许转移; (3) $M$ 审核并确认; (4) 合约收到 $C$ 发来的 token ( $D_{1}$ 提供) 后, 自动将 token $\left(D_{2}\right.$ 提 供) 发送给 $C$; (5) token ( $D_{1}$ 提供) 被自动转入销毁 地址中.

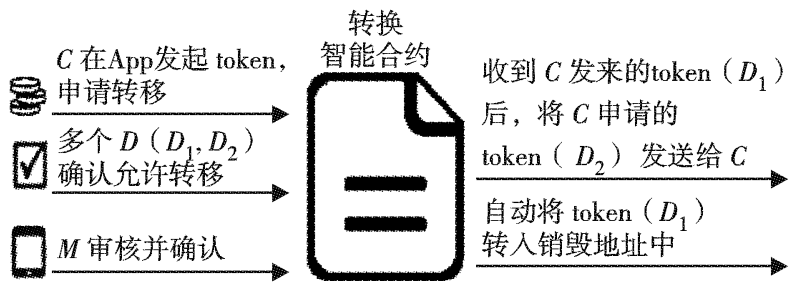

图 8 服务提供方转换链上流程

Fig. 8 Service supplier change process on blockchain

\section{4) 结算}

结算链上流程如图 9, 步骤为: (1) $D$ 发送申请 结算 token; (2) $M$ 收到申请后进行确认; (3) $D$ 收到 款项后确认; (4) token 被自动转人销毁地址.

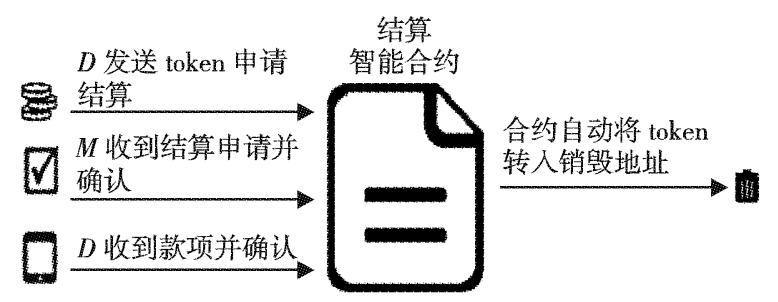

图 9 结算链上流程

Fig. 9 Accounting process on blockchain 
链下流程: (1) $M$ 收到合约请求后, 根据请求给 $D$ 发送相应款项; (2) $D$ 收到款后确认完成结算.

5）退款

退款链上流程如图 10, 具体步骤为: (1) $C$ 使用 App 发送申请退款 token; (2) $M$ 进行退款确认; (3) $C$ 收款后确认; (4) token 被自动转人销毁地址.

链下流程为: (1) $M$ 收到退款请求后, 向 $C$ 账户 转人对应的账款; (2) $C$ 收款后确认, 退款完成.

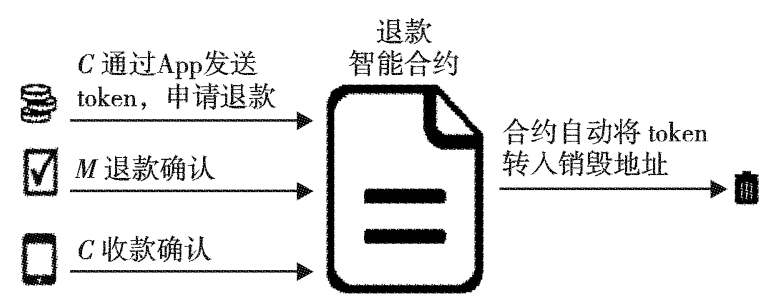

图 10 退款链上流程

Fig. 10 Refund process on blockchain

\section{3 模型优势}

1）信息透明及不可篡改, 增进互信

传统汽车售后服务预付费产品体系中, 信息的 不透明和不对称使得各方都难以维护自身的权益. 在本模型中, $M 、 D$ 和 $C$ 三方之间的交易明细都存储 在区块链上, 所有交易信息均可在链上查找验证, 且记录不可篡改, 有效保障了联盟链内部的信息透 明, 维护了各方权益.

2）提高服务质量和客户满意度

本模型支持服务商转移功能, 可激励联盟成员 提供更好的服务品质以提升竞争优势, 赢得客户, 促进客户的满意度和忠诚度的提升, 以及联盟链成 员之间的互信.

区块链技术的引入还可提高交易速度, 满足客 户对于效率的追求. 以 Hyperledger Fabric 联盟 链 ${ }^{[12]}$ 为例, 其默认每 $2 \mathrm{~s}$ 产生 1 个区块, 每个区块 10 笔交易，一天的交易量约为 18000 笔, 可以满 足现有市场的交易需求, 且区块时间可自主调节.

\section{3 联盟链代币}

\section{1 联盟链代币的构建}

\section{1.1 代币参与主体}

由汽车厂商成立相应的代币发行机构, 通过区 块链技术发行代币并推广建立相应的服务生态系 统. 发行权由汽车厂商中心控制, 使用独立的用户 身份数据保存机制, 记录用户的身份账号. 用户和
相应的服务提供商可到本机构进行代币与法定货币 的兑换，代币与法定货币保持稳定的兑换汇率. 其 他合作公司也可根据自身需要发行代币, 其他币种 可根据汇率与汽车厂商发行的代币进行兄换, 兑换 授权由汽车厂商决定.

\section{1 .2 代币的兑换}

兑换机构为汽车厂商. 兑人过程是指用户将持 有的法定货币兑换为代币. 兑出过程是指用户通过 代币兑换机构兄换成法定货币或服务. 在区块链 上, 可设置代币的兑换期限, 期限内代币只流通且 不支持兑换, 超出期限后, 允许代币兑换. 代币兑 换与法定货币保持固定汇率, 保证用户手中的代币 不会贬值. 代币兑换过程如图 11.

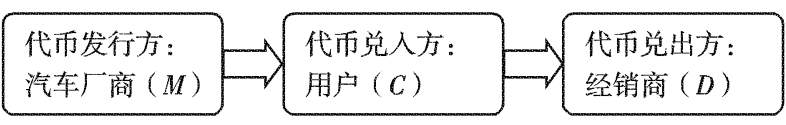

图 11 代币兑换流程

Fig. 11 Token exchange process

\section{1.3 代币的流通}

代币因为业务而产生并在交易达成后发生持有 权的转移, 由此形成了代币的流通过程. 在流通过 程中, 由于使用了区块链技术, 代币具有数据记录 功能, 不仅记录交易信息, 还记录包括用户数据等 信息, 同时根据用户意愿可在联盟链参与方之间分 享相关数据.

\section{2 代币相关流程}

联盟链的代币由汽车厂商发行或销毁, 每次发 行或销毁的代币数量均由汽车厂商指定, 代币默认 与法定货币保持固定汇率. 代币的发行和销毁流程 如图 12, 具体步骤如下.

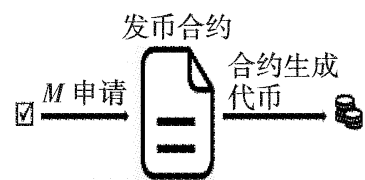

(a) 发行流程

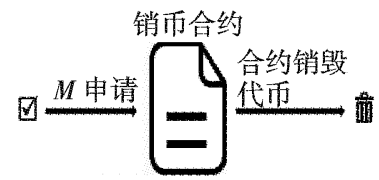

(b) 销毁流程
图 12 代币的发行和销毁流程

Fig. 12 Token issue process and destroy process

1) 代币发布流程: (1) $M$ 申请发行指定数量的 代币; (2) 合约根据指定值产生对应数量的代币.

2) 代币销毁流程: (1) $M$ 申请销毁指定数量的 代币; (2) 合约根据指定值销毁对应数量的代币.

\section{3 发行联盟链代币的优点}

由汽车厂商发行的代币, 具有以下优点. 
1) 实现汽车生态参与方的互信. 在区块链技 术背景下，建立基于信任和互利的闭环生态系统， 打造参与方彼此互信认同的价值共享平台, 能够更 加广泛地促进汽车后市场行业的健康发展, 实现良 性可持续的互惠互利，促进整个行业共同发展.

2）提高客户忠诚度. 汽车厂商通过系统平台 直接面向用户，在其产品销售过程中将具有更大的 自主性, 可为用户提供更符合需求的服务, 实现客 户长期持续的消费行为. 由于代币仅能在品牌体系 内使用，用户购人的代币也仅能在该品牌经销商处 兄出. 用户可以通过购车回馈、售后服务预付款、 用户互动等多触点购人代币, 持续在该品牌生态中 使用代币，有效维系了用户忠诚度. 根据本研究对 象内部测算，其在中国市场发行联盟链代币后 3 年 内, 由忠诚度提高所带来的额外收人高达 10 亿人 民币，其中新车销售转接率提高 $50 \%$ ，车辆保修期 外用户的保留率提高约 $30 \%$.

3) 提高系统效率, 降低运营成本. 由于系统 以代币为中心在链上交易和结算, 大大简化了运营 流程, 提高了管理效率. 据本研究对象内部测算, 其在中国市场由效率提升带来的成本降低预计为每 年 1500 万人民币左右; 就管理效率而言，全国经 销商中的财务人员、销售顾问和服务顾问等相关岗 位，可累计减少 $500 \sim 1000$ 人，可大幅提高企业的 经营效率.

\section{结语}

在汽车后市场领域采用区块链技术, 以联盟链 的方式, 搭建多样化的服务生态平台, 可在满足汽 车厂商、经销商和用户 3 方利益的基础上，有效提 高经销商的运营效率，降低汽车厂家的经营成本和 沟通成本，实现服务过程和价格的透明化，提升客 户满意度和忠诚度.

随着联盟链的不断完善，更多的服务提供商可 根据自身实际业务情况通过使用区块链技术，加人 到联盟链中, 从而形成以 “汽车” 为中心, 汽车厂 商、用户、 $4 \mathrm{~S}$ 店、独立汽车保养服务商、汽车贷款 服务商以及汽车供应链生产商等多元主体共同参与 的服务生态联盟，以持续提高汽车行业的服务品 质，保障客户权益.

基金项目: 国家自然科学基金资助项目（61771315)

作者简介：陈 洁 (1978一), 北京大学博士研究生. 研究方向: 教育经济与管理. E-mail: jie.chen@ pku.edu.cn

引文: 陈 洁, 张胜利, 王 晖. 面向汽车生态圈的区块链应
用模型 $[\mathrm{J}]$. 深圳大学学报理工版, 2021, 38(6) : 643648.

\section{参考文献 / References :}

[ 1 ] 易 锦, 张剑平. 汽车售后服务模式创新探析 [ J ]. 汽车工业研究, 2007(10): 32-34.

YI Jin, ZHANG Jianping. Analysis on the innovation of automobile after-sale service model $[\mathrm{J}]$. Auto Industry Research, 2007(10): 32-34. (in Chinese)

[2] 葛虽汉. 汽车售后服务的国内外现状及发展趋势 $[\mathrm{J}]$. 公路与汽运, 2007(6): 43-45.

GE Yinghan. Domestic and foreign status quo and development trend of auto aftersales industry $[\mathrm{J}]$. Highways \& Automotive Applications, 2007 (6): 43-45. (in Chinese)

[ 3 ] 中国消费者协会. 2019 年全国消协组织受理投诉情况 分析 $[\mathrm{EB} / \mathrm{OL}$. (2020-01-17) [2020-10-01]. http:// www. cca. org. cn/tsdh/detail/29434. html.

China Consumer Association. Analysis of complaints accepted by the National Consumer Association in 2019 [EB/OL]. (2020-01-07) [2020-10-01]. http://www. cca. org. cn/tsdh/detail/29434. html. (in Chinese)

[ 4 ] NAKAMOTO S. Bitcoin: a peer-to-peer electronic cash system [ DB/OL ]. (2008-10-31) [2019-10-02 ]. http://bitcoin. org, 2008.

[ 5 ] 张胜利, 王滔滔, 杨 晴, 等. 高性能许可公链 $[\mathrm{J}]$. 深圳大学学报理工版, 2020, 37(3): 227-233.

ZHANG Shengli, WANG Taotao, YANG Qing, et al. Permissioned public blockchain with high performance $[\mathrm{J}]$. Journal of Shenzhen University Science and Engineering, 2020, 37(3): 227-233. (in Chinese)

[ 6 ] 袁 勇, 王飞跃. 区块链技术发展现状与展望 $[\mathrm{J}]$. 自动化学报，2016，42(4)：481-494.

YUAN Yong, WANG Feiyue. Blockchain: the state of the art and future trends $[\mathrm{J}]$. Acta Automatica Sinica, 2016, 42(4) : 481-494. (in Chinese)

[ 7 ] DECKER C, WATTENHOFER R. Information propagation in the bitcoin network [ C ]// Proceedings in the 13th International Conference on Peer-to-Peer Computing. Trento, Italy: IEEE, 2013: 1-10.

[ 8 ] DEVROYE L. Universal asymptotics for random tries and PATRICIA trees $[\mathrm{J}]$. Algorithmica, 2005, 42(1): 1129.

[ 9 ] KNESSL C, SZPANKOWSKI W. Heights in generalized tries and PATRICIA tries [C]// Proceedings of the 4th Latin American Symposium on Theoretical Informatics. Punta del Este, Uruguay: Springer-Verlag, 2000 : 298307

[10] MORRISON DONALD R. PATRICIA: practical algorithm to retrieve information coded in alphanumeric $[\mathrm{J}]$. Journal of the Association for Computing Machinery, 1968, 15 (4) : 514-534.

[11] BUTERIN V. A next-generation smart contract and decentralized application platform [EB/OL]. (2014-03-29) [2020-09-29]. https://ethereum. org/en/whitepaper/.

[12] DHILLON V, METCALF D, HOOPER M. The hyperledger project $[\mathrm{M}] / /$ Blockchain Enabled Applications. Berkeley, USA: Apress, 2017: 139-149.

【中文责编：英 子】 